\title{
In Epistemic Networks, Is Less Really More?
}

\section{Sarita Rosenstock, Justin Bruner, and Cailin O’Connor*†}

We show that previous results from epistemic network models by Kevin J. S. Zollman and Erich Kummerfeld showing the benefits of decreased connectivity in epistemic networks are not robust across changes in parameter values. Our findings motivate discussion about whether and how such models can inform real world epistemic communities.

1. Introduction. Recently, philosophers of science have begun using network models to investigate the effects of communication structure on inquiry in epistemic communities. ${ }^{1}$ These models have been taken to potentially in-

Received February 2016; revised June 2016.

*To contact the authors, please write to: Sarita Rosenstock, Department of Logic and Philosophy of Science, University of California, Irvine; e-mail: rosensts@uci.edu. Justin Bruner, School of Philosophy and School of Politics and International Relations, Australian National University; e-mail: justin.bruner@anu.edu.au. Cailin O'Connor, Department of Logic and Philosophy of Science, University of California, Irvine; e-mail: cailino@uci.edu.

$\dagger$ We would like to thank Liam Kofi Bright, Remco Heesen, Bennett Holman, Aydin Mohseni, Hannah Rubin, Kim Sterelny, Jim Weatherall, and Kevin Zollman for helpful comments and feedback, as well as Jeff Barrett, Simon Huttegger, Louis Narens, Don Saari, Brian Skyrms, and the other participants in the UC Irvine Social Dynamics research group. We are also grateful for questions and comments from the audiences at the following 2016 conferences: Medical Knowledge in a Social World at UC Irvine, Models and Simulations at the University of Barcelona, the Formal Epistemology Workshop at the University of Gröningen, and the Lund Conference on Games, Interaction Reasoning, Learning, and Semantics. This material is based on work supported by the National Science Foundation under grant STS-1535139.

1. We follow previous authors by taking 'epistemic community' to refer to groups of interacting researchers such as those in academia or industry and 'network' to refer to a modeling construction in which agents are represented as points on a graph with edges denoting that the connected agents communicate with one another. For a nonexhaustive list of work in this area, see Zollman (2007, 2010, 2012, 2013), Grim (2009), Alexander

Philosophy of Science, 84 (April 2017) pp. 234-252. 0031-8248/2017/8402-0003\$10.00

Copyright 2017 by the Philosophy of Science Association. All rights reserved. 
form which communication structures among epistemic agents will be more or less successful in that they lead to correct beliefs about the world or facilitate consensus building in epistemic groups.

Some of the earliest, and most influential, models in this literature are presented by Zollman $(2007,2010)$, who argues that, under some circumstances, it will actually benefit an epistemic community to have a less connected communication network. He shows that under certain modeling conditions researchers can, by maintaining fewer lines of communication, improve the chances that as a group they eventually come to believe true things about the world. A well-networked group of researchers, however, will tend to arrive at consensus more quickly in these models but will be more prone to error.

This article will highlight some of the ways that Zollman's results, and related results from Kummerfeld and Zollman (2016), are sensitive to parameter choices. As we will argue, our exploration sharpens the original result. We find that in these models, less connectivity can improve inquiry only in a small parameter range in which learning is especially difficult: situations in which there are relatively few researchers, relatively small batches of information collected at a time, and small differences between the success of the correct and incorrect theories that the researchers are comparing. When inquiry is easier, decreased connectivity simply slows learning and provides no particular benefits.

We will argue that our results, and previous results demonstrating the sensitivity of epistemic network results to structural modeling choices, have implications for how these models should be taken to inform real epistemic communities. We think a main epistemic role such models play is in providing 'how-potentially' stories. They direct our attention to phenomena that might, potentially, occur in real communities. This can prompt further investigation, both theoretical and empirical, into such phenomena. We argue, though, that further investigation is most strongly warranted when a phenomenon is at least reasonably stable, or robust, to changes in the model (whether these are to parameter values or other structural features) or when we are reasonably confident that the conditions under which the phenomenon occurs in the model in fact obtain in the real world. Our results attenuate the degree to which these conditions hold for the case of decreased connectivity improving inquiry. (Although, as we will point out, there are some results from epistemic network models that are more strongly robust to modeling choice.) Furthermore, as we will illustrate using our modeling results, there are alternative ways to improve outcomes for epistemic communities when inquiry is difficult that do not carry the same downsides as decreased communication.

(2013), Mayo-Wilson, Zollman, and Danks (2013), Holman and Bruner (2015), and Kummerfeld and Zollman (2016). 
We start in section 2 by describing Zollman's models in detail and discussing some of the ways his results have been used in subsequent literature. We highlight, in particular, work that takes his models to potentially have implications for real epistemic communities. Section 3 presents the results of replicating Zollman's models in a wider parameter space. When one shifts the relative success rates of theories in Zollman's models, or when different assumptions are made about the size of epistemic networks, his original results do not hold. In section 4 we look at a similar model from Kummerfeld and Zollman (2016) and show that, here too, differences in network performance disappear under changes in parameter settings. We conclude by discussing how model robustness in these cases should be taken to inform the relevance of network epistemology models to the real world.

\section{Previous Models, Results, and Impact}

2.1. Models. The model used in Zollman $(2007,2010)$ was first introduced by Bala and Goyal (1998). These authors consider the general conditions under which a network of learning agents will adopt the same beliefs and the conditions under which these learned beliefs will be successful. We will describe the exact version of the model employed by Zollman (2007) and then the version employed by Zollman (2010).

To motivate the model, consider the following situation. A group of clinicians all use drug $\mathrm{X}$, with a known success rate, to treat a certain disease, but a pharmaceutical company has recently released drug $\mathrm{Y}$, with an unknown success rate, for the same purpose. Each clinician has some belief, based on previous experience with drug $\mathrm{X}$ and new data on drug $\mathrm{Y}$, as to which is more successful. Clinicians begin to prescribe the drug they prefer, all the while noting the success rates of the new drug. At the same time, clinicians communicate with colleagues about their practices and so gain information about the new drug's success that way as well. If, as a result of this information, a clinician becomes convinced that she is doing the wrong thing, she will switch drugs. In a setup like this, the agents will learn from their neighbors, as well as from their own choices, which action to take in the future. As such, the structure of their communication network may influence the outcome of their inquiry.

This sort of scenario can be modeled using what is called a "two-armed bandit" model as follows. Imagine a network of agents who may take one of two actions, A or B. A yields a good outcome with probability $p_{\mathrm{A}}=0.5$. Action B yields a good outcome with probability $p_{\mathrm{B}}=0.5+\phi$ where $\phi \in[0,0.5]$. However, agents do not have full information about the success of action B. They believe that it is successful either with probability $0.5+\phi$ or with probability $0.5-\phi$. In other words, they are unsure about whether this action is better or worse than $\mathrm{A}$. 
Agents in this model start with some randomly chosen belief about whether $\mathrm{A}$ or $\mathrm{B}$ is preferable (whether $p_{\mathrm{B}}=0.5+\phi$ or $p_{\mathrm{B}}=0.5-\phi$ ). This belief is modeled as a number between 0 and 1 . For example, an agent might have a degree of belief of 0.8 , meaning that she thinks there is a 0.8 probability that $\mathrm{B}$ is the better action (that $p_{\mathrm{B}}=0.5+\phi$ ). Agents then repeatedly choose which action to take. In each round, agents use their degree of belief to choose an act. If it is less than 0.5 , they choose A; if it is greater than 0.5 , they choose B.

Upon making a choice in each round, an agent is rewarded with a payoff selected from a binomial distribution of parameters $p$ and $n$. What this means is that in a single round an agent will select her preferred action $n$ times, and each time she will get a payoff of ' 1 ' with probability $p$. For example, suppose that $p_{\mathrm{B}}=0.65$, and an agent chooses action $\mathrm{B}$ in a round. If $n=100$, that agent will pick B 100 times, each time receiving ' 1 ' with probability 0.65 .

After acting, agents observe their outcomes and the outcomes of neighbors in their network. They use Bayesian updating to alter their degrees of belief about $\mathrm{A}$ and $\mathrm{B}$ on the basis of the success rates of these actions. This sort of updating involves the application of Bayes's rule to an agent's prior degree of belief, using all the evidence gathered by an agent and her neighbors.

Upon simulating this model, agents in a network will tend to slowly converge to the same action. In other words, all agents will learn to choose A or all agents will learn to choose B. Zollman (2007) ran simulations of this sort, ending the simulations either when all agents chose A (thus ending the acquisition of new information that might lead them to choose B) or when all agents had a very high degree of belief that B was the better action (making their reversion to A vanishingly unlikely).

Zollman (2010) employs a slightly more sophisticated version of this model. In this version, agent beliefs are modeled using what is called a beta distribution. This is a distribution on $[0,1]$ where the value of the distribution at a point represents the agent's belief that that number is the real success rate of choice $\mathrm{B}$. At the beginning of a simulation, agents are given a random beta distribution with parameters $\alpha$ and $\beta$ chosen from the interval $[0,4]$. These parameters determine the shape of the distribution, although it is not important for our purposes to understand the details of how they do so. In each round of simulation, these agents then use their distribution to decide which action they think will be better. After acting, as before, they use Bayesian updating to alter their distributions on the basis of their outcomes and the outcomes of their neighbors. Zollman (2010) runs these simulations for 10,000 rounds before stopping them to see what agents have learned.

2.2. Zollman's Results. The central result of Zollman (2007), which is replicated in Zollman (2010), is that the network structure in these models 
influences inquiry in significant ways. In particular, Zollman argues that a sparser network structure can benefit an epistemic community. More connected networks converge to uniform beliefs more quickly, but sparse networks are more likely to converge to the true belief.

Zollman (2007) achieves these results by running the models described above for agents in different network structures. First he considers three networks with 3-10 agents - the cycle, the wheel, and the complete graph. Figure 1 shows these three types of networks (with eight agents in this case). He also considers every possible network formation with six agents. Figure 1 also displays two examples of six-agent network formations. For both sets of networks, and for both types of models, Zollman finds that more connections speed the learning of the community but decrease the chances that the community learns to take the better action.

Why these effects? As Zollman argues, in highly connected networks, runs of unlucky results can spread quickly and convince an entire community to start taking the wrong action. In other words, the group can too quickly learn the wrong thing. In sparser networks, such results are only seen by a few agents, so a single unrepresentative batch of data cannot simultaneously convince the entire community to perform the inferior, uninformative action. It is more likely that a few agents will maintain the better belief and thus enable their neighbors to learn from their continued data collection. In a real epistemic community, this translates to pockets of interacting

A
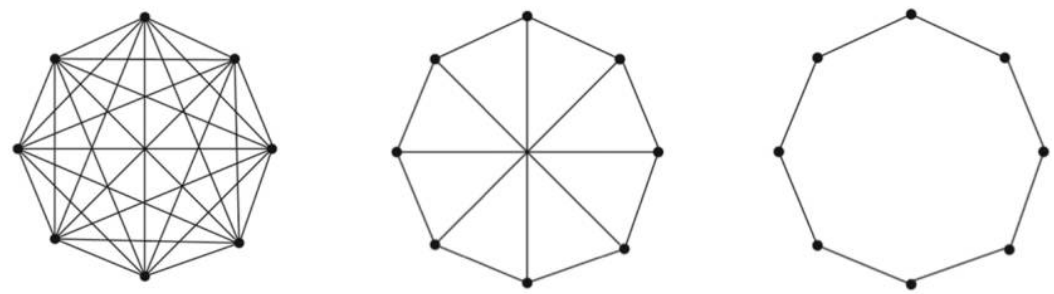

B
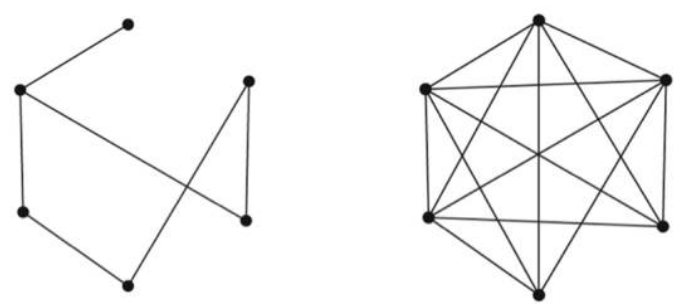

Figure 1. Various network formations. $A$, an eight-agent network with complete graph, wheel, and cycle configurations respectively; $B$, two six-agent networks, one more sparse, the other more connected. 
researchers who may maintain different sets of beliefs but eventually share successful ones.

Zollman (2010) provides extended results using the second class of models. He shows that, in particular, it benefits communities of epistemic agents to hold a diversity of opinions for some period of time. In sparse networks, this is achieved because agents are less likely to change each others' minds quickly. It can also be achieved, Zollman argues, by ensuring that agents begin simulations with more extreme beliefs, which are harder to shift.

2.3. Impact. Zollman $(2007,2010)$ has been widely cited both in philosophy and the social sciences. In this section, we motivate the claim made in the beginning of the article that the best way to understand the main epistemic role of his work is as 'how-potentially' modeling. His results have been taken, in a number of cases, to suggest that a sparse network structure in epistemic communities may indeed potentially help promote successful inquiry. Our intent here is to underpin the importance of discussing the robustness of said results and the application of these results to real world communities.

'How-possibly' models have received a great deal of attention from philosophers of science. These models typically work by showing that some phenomenon can possibly result from (often surprising) preconditions. For example, Quine $(1936,2013)$ famously argued that without established linguistic convention, it is impossible to assign meaning to terms. Skyrms's (2010) work on the evolution of signals showed that, contra Quine, conventional meaning can emerge through simple processes of learning and evolution. In other words, in answer to the question, is it possible to generate linguistic convention without existing linguistic convention? Skyrms's models provide a definitive yes. When it comes to how-possibly modeling of this sort, there is no need to ensure that modeling results are robust. All that is required from the model is evidence that a phenomena can occur under the right conditions, not that this phenomena is likely or that it is, in fact, one we might see in the real world.

Zollman's models do not fall under the heading of how-possibly modeling. Neither, though, are they so clearly representative of real world communities that they can be taken as directly informative of these communities. Rather, they present a phenomenon that has the potential to occur in a real epistemic community. In doing so, they warrant or direct us toward further research in this area. Their interest to philosophy of science has been largely in this vein.

To support this claim, we provide a few quotes illustrating the way Zollman and others have discussed these models. Zollman (2007), for example, concludes by saying that "this model suggests that in some circumstances there is an unintended benefit from scientists being uninformed about exper- 
imental results in their field.... When we want accuracy above all else, we should prefer communities made up of more isolated individuals" (586). Zollman (2010) concludes with, "Looking at these scientists from the perspective of individualistic epistemology, one might be inclined to criticize the scientists' behavior. However, when viewed as a community, their behavior becomes optimal" (33). In these quotes he describes the results as applying to 'scientists' and 'communities', entities from real epistemic groups. And the work is not described as how-possibly modeling, that is, to disprove a claim like 'communication always improves science'.2

Others have echoed this move. With respect to the result that less connectivity may help a research community, Strevens (2010) writes: "It might, for example, be preferable for scientists not to take into account too much information about their colleagues' beliefs about a problem, if a few authoritative pronouncements would stifle much-needed diversity in the range of approaches to a problem.... The question how to tune attention to authority in the short term so as to find a level of diversity that maximizes correctness in the long term has been explored with considerable insight by Zollman (2007)" (20). Wray (2013) reports that "Zollman found that full communication in a research community is sub-optimal, as lines of communication in a group not only aid in the spread of truth, but also facilitate the spread of errors" (78).

As to the related result that diversity of beliefs among agents can promote inquiry, Douven and Kelp (2011) say that “Zollman's intriguing work shows that, from a socio-epistemic perspective, it may be important to maintain epistemic diversity in a community of agents, at least for a while, and that, for that reason, it is not always best if all agents have access to all information available in their community; for the same reason, a certain dogmatism on the part of the agents ... may be beneficial" (277). Muldoon (2013) writes that "Zollman showed how transient diversity in beliefs, whether fostered by limited communication or stubborn scientists, can help ensure that scientific consensus tracks the truth" (123).

In the quotes above, authors describe these models as potentially informing 'scientists' and 'communities'. Our goal here is not to claim that these authors are making any sort of mistake in describing Zollman's results as they do. We are simply pointing out that the models are taken as potentially telling us something about the real world, rather than showing that some phenomenon is possible in principle. The question arises then: how seriously should we take these results? Should they point us to further research in this area? Should they increase our confidence that communication might lead to

2. Personal correspondence with Zollman confirms that, indeed, he is thinking of the epistemic role of these models in approximately this way. 
poorer inquiry? In the next sections we analyze the robustness of Zollman's findings with an eye to these questions.

3. Expanding the Parameter Space. In what follows, we present the results replicating Zollman's (2007) simulations with a wider parameter space. We find that parameters for which there is a notable benefit to decreased network connectivity occupy a relatively small niche of the total space.

We ran Bala and Goyal style simulations varying $p_{\mathrm{B}}$ (the success rate of the better action), $n$ (the number of trials an agent performs each round), network configurations, and network sizes. ${ }^{3}$ For each parameter setting, we ran at least 10,000 trials and checked to see whether each had converged to the favorable outcome (and how quickly). We focused on two network configurations - the cycle and the complete graph. We chose these configurations as representative of highly dense and sparsely connected networks and because both were considered in Zollman's original paper. Where his results occur, they ought to occur for these two networks. We also ran similar simulations mimicking the models used in Zollman (2010) but for smaller sets of parameter values. The results of these simulations were similar in all cases to those we present in more detail here.

We refer to the positive difference between successful convergence in the cycle network versus the complete network as the "Zollman effect" for the rest of the article, as this measurement represents for these simulations the general effect noted by Zollman - that sparser networks are more reliable than well-connected ones.

3.1. Success Rates. In Zollman $(2007,2010)$, the probability that action $\mathrm{A}$ is successful is always $p_{\mathrm{A}}=0.5$. The probability that action $\mathrm{B}$ is successful is always chosen to be $p_{\mathrm{B}}=0.501$. This means that in models from Zollman (2007), agents believe that B has a success rate of either 0.501 or 0.499 .

Obviously, 0.5 and 0.501 are very close values. In this section, we show that in many cases as $p_{\mathrm{B}}$ increases by even a relatively small amount, the Zollman effect vanishes. The intuitive explanation for this is that when $p_{\mathrm{B}}$ is higher, trials of action A and action B are less likely to have similar results, so agents have an easier time distinguishing them. As a result, a sparse net-

3. We ran simulations for a variety of parameters, with $p_{\mathrm{B}}$ ranging from 0.501 to $0.7, n$ ranging from 1 to 6,000 , and network size ranging from 4 to 100 . We were constrained by computing power in some cases. For example, lower values of $n$, and $p_{\mathrm{B}}$, cycles and larger networks took longer to run. Generally, though, trends we noted held for all parameter values explored. Results reported here are winnowed from these, but the trends should be thought of as generally applicable. Recorded results and the python code used to generate them can be found in the online edition. 
work structure does not help the community learn correct beliefs but still slows learning significantly.

Figure 2 shows outcomes of our simulations when we vary $p_{\mathrm{B}}$ but keep population size and $n$ fixed at values considered in Zollman's original models, 10 and 1,000 respectively. The $x$-axis tracks the value of $p_{\mathrm{B}}-0.5$, the differential success rate for the better action. The $y$-axis shows, for the complete and cycle networks, how frequently they converge to the successful action.

As discussed, as $p_{\mathrm{B}}$ increases, the Zollman effect decreases. In other words, the difference in likelihood of successful convergence between the two network configurations decreases. This difference is 0 for $p_{\mathrm{B}} \geq 0.525$. For $p_{\mathrm{B}}=0.51$ there is a difference of less than $2 \%$ in the successful convergence rates. This occurs because, as $p_{\mathrm{B}}$ increases, all networks are able to successfully converge to correct beliefs with very high probability, so sparse networks are not particularly helpful.

Zollman (2010) says, with respect to the Zollman effect and different values of $p_{\mathrm{B}}$, "The degree of difference here should not be taken too seriously; it can be altered by modifying the difference in objective probabilities of the different methodologies. However, the ordering of the graphs remains the same - the cycle is superior to the wheel which is superior to the complete graph" (17). Zollman (2007) includes the following footnote, "The results for both reliability and speed are robust for [the cycle, wheel, and complete networks] across . . . difference in payoff between the good and uninformative actions. Although these different modifications do effect the ultimate speed and reliability of the models, for any setting of the parameters the relationship between the three networks remains the same" (n. 7). His claim is that the effect occurs, to varying degrees, for all parameter values in these models. Given our findings, we think it more accurate to

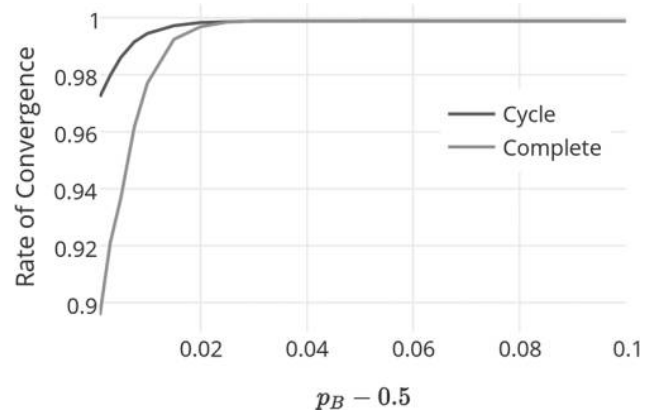

Figure 2. Simulation results showing the rates of convergence (i.e., portion of our simulations for which the agents converged to the better action) as a function of $p_{\mathrm{B}}$ for the complete and cycle networks of size 10 with $n=1,000$. Color version available as an online enhancement. 


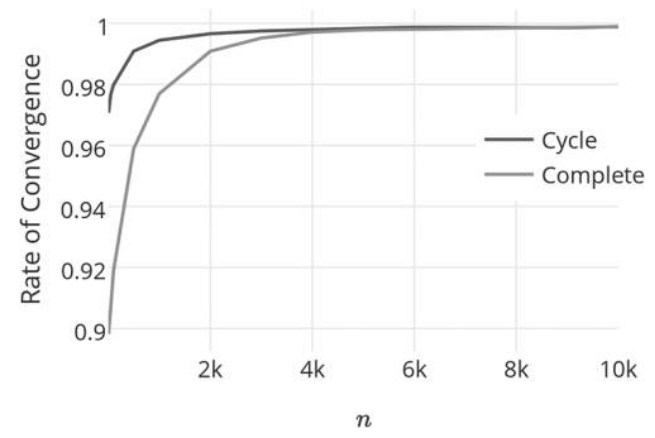

Figure 3. Simulation results showing the influence of $n$, or the number of trials per round, on convergence for complete graphs and cycles for a population of size 10 with $p_{\mathrm{B}}=0.51$. Color version available as an online enhancement.

say that the effect does not arise for significant portions of the relevant parameter space. Note, though, that we never see the effect reverse so it may be that it holds but to such a slight degree that it is undetectable given the limits of our computational setup.

3.2. Number of Trials per Round. Figure 2 uses Zollman's choices of $n=1,000$ for the number of times per round that each agent tries her preferred action. In expanding the parameter space investigated for these models, we found that decreasing $n$ actually increases the Zollman effect across models. Figure 3 shows the simulation results for a population of size 10 with $p_{\mathrm{B}}$ held fixed at 0.51 , where $n$ ranges from 10 to 10,000 . As this figure shows, the difference in rates of successful convergence for the cycle and complete networks is more significant for lower $n$.

In these simulations, for larger $n$, probabilistically it is more likely that the data obtained by each actor will reflect the true underlying probabilities of success for each action, and ditto for the combined information between actors. For smaller $n$, actors are more likely to receive strings of data where the less preferable action is successful, or the preferable one is unsuccessful, as a result of the law of small numbers. In a situation like this, network sparsity helps the entire community avoid these misleading strings. ${ }^{4}$

4. Note, holding fixed other parameters and varying $n$, actors must receive about the same number of data points to converge on a set of beliefs. So, e.g., when $n=20$ and actors are in a cycle, they receive 60 data points each round. If $n=100$, they receive 300 data points each round. If the $n=20$ network converges in 20 rounds on average (1,200 data points total for each agent), the $n=100$ network will converge in about 12 rounds on average ( 1,200 data points total for each agent). This supports our claim that the difference in the Zollman effect across these simulations is indeed due to how often they update and, in particular, the fact that in low $n$ simulations actors update more frequently and see misleading short strings of data. 
3.3. Number of Agents. We also looked at larger populations than Zollman considered in his original papers. In these larger populations, we found that the significance of the Zollman effect, again, decreased. Once more, we ran simulations of complete and cycle networks for various parameter values and measured what proportion of simulations (again, out of at least $10,000)$ converged to the better action.

Figure 4 shows results of these simulations. For this figure we look at the Zollman effect for the parameters $n=1,000$ and $p_{\mathrm{B}}=0.501$, matching Zollman's original models. The Zollman effect is strongest for smaller networks, but as network size increases it drops off. We find similar results for other parameter values as well. Note that while in this figure the effect occurs at all values of $n$, this is not so for many other parameter values.

What is going on in this case? For these larger networks, the effects of connectivity are relatively unimportant because actors are able to gain so much information about their target inquiry from their larger cohort. Thus in almost every case, these larger networks converge to correct beliefs about the world. This ends up meaning that sparser networks do not give agents a notable advantage in terms of accuracy.

Again, sparse networks tend to learn slowly, and this is especially the case for large networks. Figure 5 shows the difference in speed of convergence on average for cycle versus complete networks. The cycle is always a slower learner, and this speed difference increases as population size increases, although the cycle's advantage in rate of convergence diminishes. This makes sense, as in larger networks the tendency to perform action A takes longer to propagate along a cycle, and the difference in connectivity between cycle and complete networks is much larger. Thus as network size increases, the potential benefit of decreased communication goes down, and the cost (i.e., speed of convergence) associated with reducing network con-

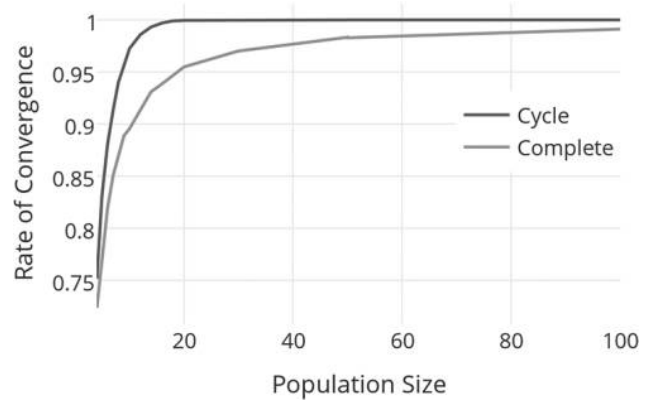

Figure 4. Rate of convergence by population size for complete graph and cycle, $p_{\mathrm{B}}=0.501, n=1,000$. Color version available as an online enhancement. 


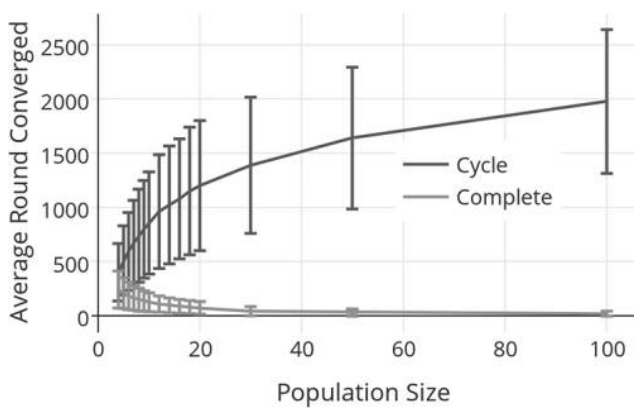

Figure 5. Speed of convergence in cycle and complete networks by population size, $p_{\mathrm{B}}=0.501$. Vertical bars show 1 standard deviation. Color version available as an online enhancement.

nectivity climbs dramatically. For a population of 100 agents, the convergence rate for the complete network is $99.12 \%$, versus $100 \%$ for a cycle, while the average round at which the community achieves convergence is 20 for the complete network and 1,977 for the cycle.

One argument Zollman (2007) gives is that there is a trade-off, in such networks, between learning quickly and learning well. More connected networks learn quickly but may fail to converge to correct beliefs, while sparser networks are more accurate but slower. When the Zollman effect is nonexistent, this trade-off, of course, also disappears. Sparse networks still learn more slowly than densely connected ones, but there is no particular benefit from sparse network structure.

To drive the point home, we include one more figure. Figure 6 shows the Zollman effect as a function of both the difference between the success rates of the two theories and population size for $n=1,000$. As is evident from this figure, which only shows $p_{\mathrm{B}}$ up to 0.7 , for the majority of the parameter space there is no effect.

4. Exploratory Agents. In this section, we briefly turn our attention to models from Kummerfeld and Zollman (2016) who find a similar relationship between connectivity and successful inquiry in structurally different models than those we have been considering. They also show that when agents have more exploratory tendencies, complete networks are more successful than cycles (a sort of reverse Zollman effect). We will show that the effects in Kummerfeld and Zollman (2016), like those in Zollman (2007, 2010), disappear under broader parameter values.

Kummerfeld and Zollman (2016) look at models that are very similar to those discussed here. Agents are once again presented with two actions. 


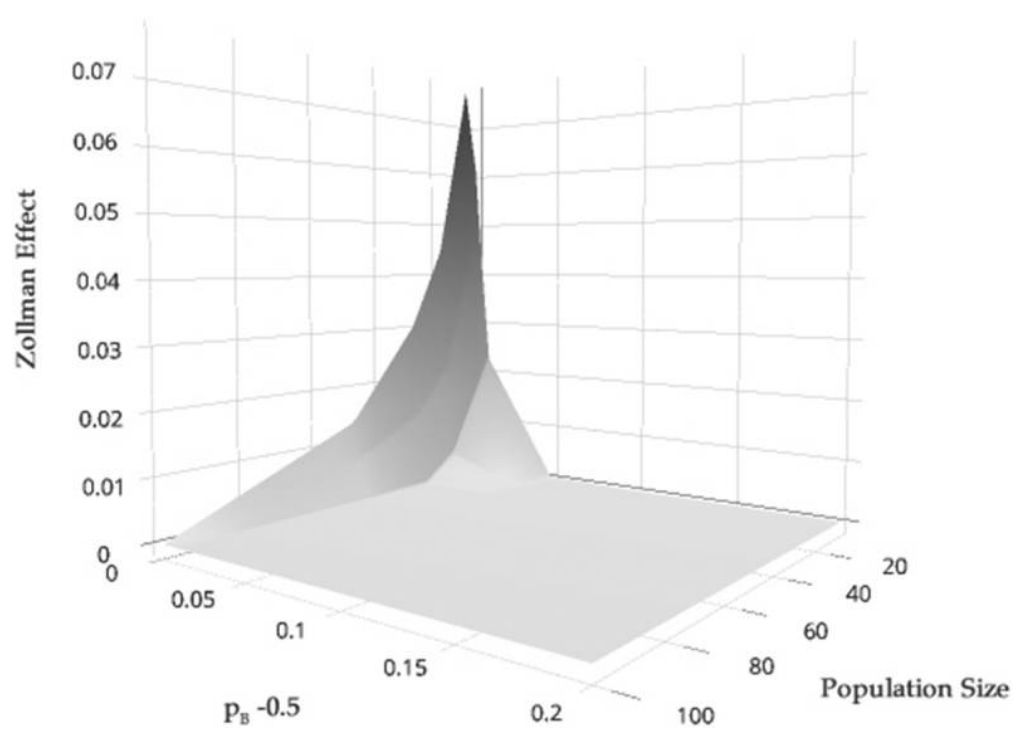

Figure 6. Zollman effect as a function of $p_{\mathrm{B}}$ and population size, $n=1,000$. Color version available as an online enhancement.

They are part of eight-agent either complete or cycle networks. The agents in these networks, however, are not Bayesian updaters. Rather, they play a strategy called $\varepsilon$-greedy. This means that each agent calculates, on the basis of her observations and those of the agents she is connected to, which action has had the highest average payoff in the past. She takes this action in the next round with probability $1-\varepsilon$ and explores, or tries the other action, with probability $\varepsilon$. Unlike the agents in the models we have considered thus far, then, these agents do not always pick what they think is best. Instead they explore all options, with $\varepsilon$ determining how likely they are to be exploratory.

The payoffs of the two actions in these models also work differently from those of the previously discussed Zollman models. The worse action always yields the same outcome of 0 . The better action is also the riskier one. It yields an outcome drawn from a normal distribution centered on a value greater than 0 .

Kummerfeld and Zollman (2016) find that for low $\varepsilon$ (the situation closest to the Zollman [2007, 2010] models), cycle networks are more successful than complete networks in that, on average over all rounds and all agents, the payoff is higher. Note that this is a different measure of success than we have been discussing but one that also tracks whether agents are taking the better action. As $\varepsilon$ increases, though, complete networks start outperform- 
ing cycles. In other words, as agents explore more on their own, connectivity starts to be beneficial to inquiry.

We looked at an expanded range of parameter values for these models and, again, found that network effects disappeared for wider parameter values. Kummerfeld and Zollman (2016) looked at models in which the better action resulted in a payoff drawn from a normal distribution centered on 1 with a standard deviation of 9 . We explored a much less extensive parameter space for these models than the Bala and Goyal style ones, but we looked at models in which this distribution instead had a mean $=1,2,3$, and 4 and standard deviation $=3$ and 9 . We considered a version with more exploratory agents, $\varepsilon=0.35$, where the complete network was better, and less exploratory agents $\varepsilon=0.05$, where the cycle network was more successful instead. ${ }^{5}$

We found that as the mean of the payoff distribution increased, network effects on performance decreased for both low and high $\varepsilon$. We also found that decreasing the standard deviation of the distribution decreased network effects. Figure 7 shows these results. The $x$-axis represents the mean of the payoff distribution from 1 to 4 . The $y$-axis tracks the difference in success between the cycle and complete network for each parameter value. As the mean increases, the difference between the two networks decreases for all parameter values. Also, as the standard deviation decreases, so does the difference in success. We confirmed this for other parameter values with networks of size 7 using fewer rounds (to decrease computational requirements). For these networks again, increasing the mean and decreasing the standard deviation of the payoff distribution decreased network effects for both low and high $\varepsilon$.

Once again, we find that in the larger parameter space of this network epistemology model, the effects of connectivity disappear over many values. In this case, we see both the disappearance of a Zollman effect and a sort of reverse Zollman effect where connectivity improves inquiry. Notice that in these simulations again, when agents have an easier time determining the better action - when the mean is higher and the standard deviation lower-network structure is less important.

5. Discussion. In this section, we first pull out some relevant lessons from our extended analysis of the models in Zollman $(2007,2010)$ and Kummerfeld and Zollman (2016). We then turn to the implications of this work, and

5. We looked at networks of size 8 . Each simulation was run for 1,000 rounds. In each round, actors performed the action they thought best 50 times, with probability $1-\varepsilon$. We ran 100 simulations at each parameter value. The reported success rates are the average payoffs for all actors over all rounds of simulation. 


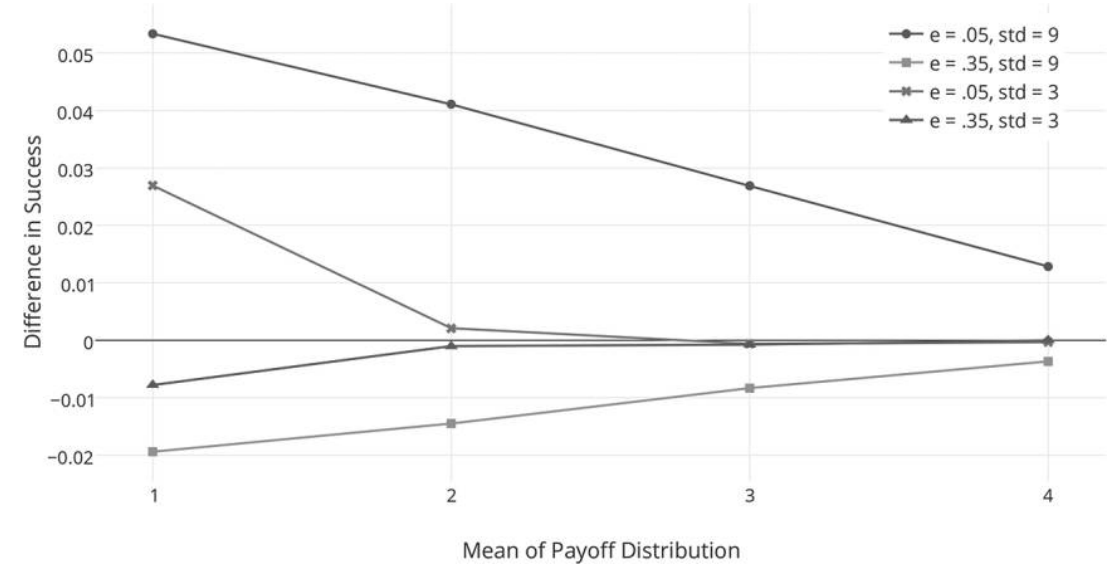

Figure 7. Difference in performance between cycle and complete networks as the mean of the payoff distribution for the better action increases. Color version available as an online enhancement.

previous work, on the usefulness of epistemic network models to informing real world epistemic communities.

5.1. When Does Network Structure Matter in the Models? In Bala and Goyal style models of epistemic networks, less connectivity can improve the accuracy of learning in the long term, but this only happens for certain areas of parameter space. In particular, the effect occurs under circumstances in which learning is more 'difficult' for the agents in that the community has more trouble distinguishing between two alternative theories.

The learning situation can be more difficult in this way when the parameters have the following features:

1. The two actions that agents may take are more similar in terms of success rates (low $p_{\mathrm{B}}$ );

2. The population size is smaller; and

3. The amount of data collected each round is smaller (low $n$ ).

In the first case, more data are necessary to accurately determine which action is the preferred one. In the second and third cases, agents receive less data each round. In all three cases, (relative) sparsity of data allows for misleading strings of information to sway epistemic communities to incorrect beliefs, meaning that there is an opportunity for network structure to influence inquiry.

To illustrate this claim consider figure 8 . For each set of parameters $-n$, network size, and $p_{\mathrm{B}}-$ we average convergence times for the cycle and wheel 


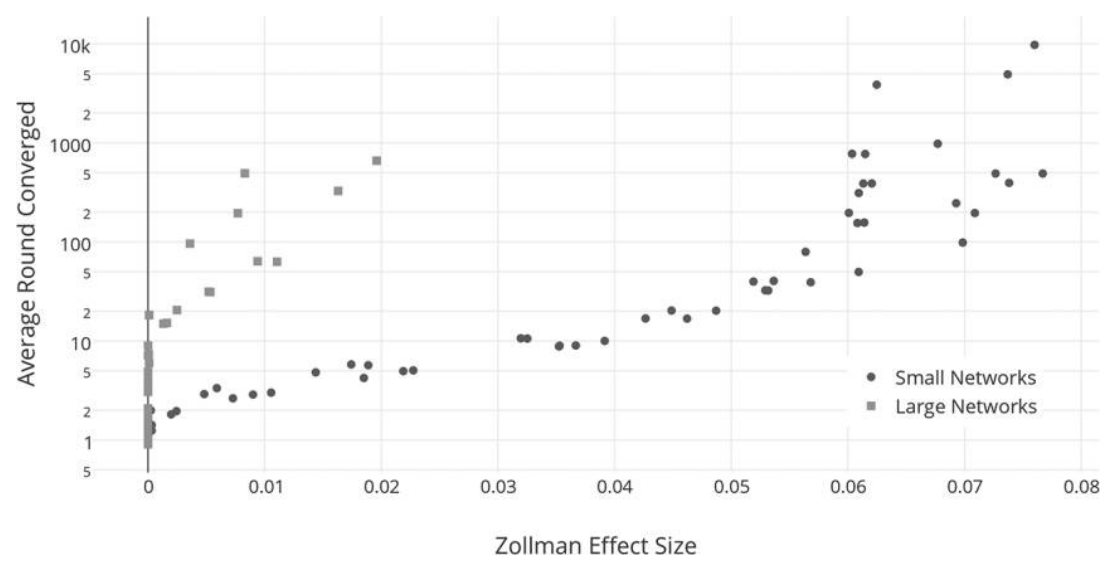

Figure 8. Average speed of convergence over cycle and complete networks as a function of Zollman effect size. Color version available as an online enhancement.

network using our data from section 3. This average gives us a proxy for how difficult it is for networks of agents to converge under each set of parameter values. We then compare this measure of speed to the strength of the Zollman effect. The data are divided into smaller networks $(6,10$, and 20$)$ and larger ones $(50,100)$ since there is a significant difference in the strength of the effect in these two sets of networks. Note that the $y$-axis is on a logarithmic scale to make the trend more visible. As is evident, there is a clear correlation between networks that take more time to converge and those where connectivity matters. This supports the claim above that network structure matters more when inquiry is trickier.

There is something unintuitive about this observation. When good information is harder to come by, this is exactly the situation in which, for these models, it is potentially useful to decrease the amount of information flowing between agents at each time step. The way to think about this is to observe that sparsity in epistemic networks can provide the very benefit that Zollman outlined (helping groups of agents to avoid preemptively converging to incorrect beliefs about the world), but it will only provide it when agents are already in a more difficult situation for inquiry. When agents have enough good data, decreasing connectivity only slows learning without providing any benefit.

In Kummerfeld and Zollman (2016) type models, again, network effects only show up when it is more difficult for agents to distinguish between alternative theories. When the mean of the payoff distribution for the more successful action is similar to the payoff for the unsuccessful action, both a Zollman effect and a reverse effect are seen for different levels of exploration. As this mean becomes larger (i.e., the better action is more obviously 
better), the different networks become equally successful. When the standard deviation of the payoff distribution is large - when it is harder to determine that the better action is, in fact, better - again both sorts of network effects are seen. And as standard deviation decreases, networks matter less.

5.2. Real World Implications. As argued previously in the article, these sorts of epistemic network models can, and have, been employed as tools that outline effects that might potentially occur in real world communities. Now we ask: should we take the potential for decreased connectivity to improve community outcomes seriously as a result of these modeling results? Do these results mean that further effort should be expended on this potential effect?

First, obviously, our results imply that we should only be looking for this effect in certain types of epistemic communities - those where inquiry is more difficult for various reasons. Previous modeling work has, similarly, shown that different epistemic situations correspond to different optimal network structures (Zollman 2013). We see this with the Kummerfeld and Zollman (2016) results, where in exploratory communities, connection improves inquiry. Likewise, Holman and Bruner (2015) look at Bala and Goyal style networks where 'biased' agents simply attempt to sway the scientific community to their views. Again, in these models connected, rather than less connected, networks are more successful.

We also think that our results should decrease confidence, at least to some degree, that epistemic network effects of the sort observed in these models regularly happen in real world communities. Why? We do not have a good sense of which real world communities are well represented by which epistemic network models. This is because these models are highly simplified. They abstract away from many relevant details of such communities. This allows them to provide an explanatory clarity that might be absent from more detailed models, but it also means that the model-world match is loose at best. As a result, we cannot say with confidence that we expect real world epistemic communities to generally fall under the area of parameter space where the Zollman effect occurs. We are unsure whether they correspond to this area of parameter space, or some other area, or some other models with different assumptions. Our results, and previous results showing the sensitivity of epistemic network effects to structural changes, should then attenuate the degree to which we worry about network effects in real epistemic communities. We do not mean to make an overly strong statement here. This is especially the case because there is at least one other model that finds a Zollman-like effect under some structural changes (Grim 2009) and two experiments with some evidence for a similar effect (Mason, Jones, and Goldstone 2008; Jönsson, Hahn, and Olsson 2015). But because we have identified many conditions under which the Zollman effect does not occur, 
and because we think many epistemic communities might fall under these conditions, our confidence in the importance of the effect decreases to some degree.

On the flip side, highly robust phenomena are more likely to have applicability to the real world because the conditions under which they occur are more likely to hold. ${ }^{6}$ When epistemic network effects are highly robust, it makes sense to take them more seriously as important findings for real world communities. There are a few results from investigations of epistemic network models that do seem robust in this way. For example, the observation by Zollman (2010) that transient diversity of beliefs is necessary to good scientific practice holds for all the models discussed. Our work suggests another possibility - that network structure matters only when inquiry is more difficult. Suggestively, Mason et al. (2008) find that when experimental subjects are tasked with searching a space for areas that yield high payoff, less connected networks seem to do better for more difficult searches.

There is one last thing to mention about the suggestion that, in some cases, it might improve inquiry to decrease communication among scientists. As noted in the last section, there is something unintuitive about the idea that in just those cases in which good data are hard to come by - small communities, small samples, theories that are hard to differentiate in terms of quality - agents should decrease the amount of data flowing between them. In fact, there are better solutions, in these cases, to the problem. More stubborn or exploratory agents, who keep investigating both theories, even if one seems less promising, will not preemptively settle on a poor theory. Standards for the amount of data necessary to confirm or deny a theory, such as those being implemented in the social sciences that face replicability crises, will likewise do the trick. These measures ensure that enough data go into each scientist's judgment that a theory is or is not the best available one, while simultaneously allowing each scientist to take advantage of all the data available. In other words, there are ways to prevent preemptively settling on a poor theory apart from ignoring good data.

\section{REFERENCES}

Alexander, J. M. 2013. "Preferential Attachment and the Search for Successful Theories." Philosophy of Science 80 (5): 769-82.

Bala, V., and S. Goyal. 1998. "Learning from Neighbours." Review of Economic Studies 65 (3): 595-621.

6. Unsurprisingly, philosophers of science disagree about the role of robustness analysis in modeling. Some maintain that robust results are better supported (Levins 1966; Weisberg 2006; Wimsatt 2012; Heesen, Bright, and Zucker 2014). Others hold that robustness analysis, in fact, cannot support modeling results (Cartwright 1991; Orzack and Sober 1993; Odenbaugh and Alexandrova 2011). We side with the former. 
Cartwright, N. 1991. "Replicability, Reproducibility, and Robustness: Comments on Harry Collins." History of Political Economy 23 (1): 143-55.

Douven, I., and C. Kelp. 2011. "Truth Approximation, Social Epistemology, and Opinion Dynamics." Erkenntnis 75 (2): 271-83.

Grim, P. 2009. "Threshold Phenomena in Epistemic Networks." In AAAI Fall Symposium: Complex Adaptive Systems and the Threshold Effect, 53-60. Menlo Park, CA: AAAI.

Heesen, R., L. K. Bright, and A. Zucker. 2014. "Vindicating Methodological Triangulation." PhilSci Archive. http://philsci-archive.pitt.edu/11158/.

Holman, B., and J. P. Bruner. 2015. "The Problem of Intransigently Biased Agents." Philosophv of Science 82 (5): 956-68.

Jönsson, M. L., U. Hahn, and E. J. Olsson. 2015. "The Kind of Group You Want to Belong To: Effects of Group Structure on Group Accuracy.” Cognition 142:191-204.

Kummerfeld, E., and K. J. S. Zollman. 2016. "Conservatism and the Scientific State of Nature." British Journal for the Philosophy of Science 67 (4): 1057-76.

Levins, R. 1966. "The Strategy of Model Building in Population Biology." American Scientist 54 (4): 421-31.

Mason, W. A., A. Jones, and R. L. Goldstone. 2008. "Propagation of Innovations in Networked Groups." Journal of Experimental Psychology: General 137 (3): 422-33.

Mayo-Wilson, C., K. Zollman, and D. Danks. 2013. "Wisdom of Crowds versus Groupthink: Learning in Groups and in Isolation." International Journal of Game Theorv 42 (3): 695-723.

Muldoon, R. 2013. "Diversity and the Division of Cognitive Labor." Philosophv Compass 8 (2): $117-25$.

Odenbaugh, J., and A. Alexandrova. 2011. "Buyer Beware: Robustness Analyses in Economics and Biology." Biology and Philosophy 26 (5): 757-71.

Orzack, S. H., and E. Sober. 1993. "A Critical Assessment of Levins's The Strategy of Model Building in Population Biology (1966)." Ouarterly Review of Biology 68 (4): 533-46.

Quine, W. V. O. 1936. "Truth by Convention." In Philosophical Essays for A. N. Whitehead, ed. O. H. Lee. London: Longmans, Green.

- 2013. Word and Object. Cambridge, MA: MIT Press.

Skyrms, B. 2010. Signals: Evolution, Learning, and Information. Oxford: Oxford University Press.

Strevens, M. 2010. "Reconsidering Authority: Scientific Expertise, Bounded Rationality, and Epistemic Backtracking." In Oxford Studies in Epistemology, vol. 3, ed. T. S. Gendler and J. Hawthorne. Oxford: Oxford University Press.

Weisberg, M. 2006. "Robustness Analysis." Philosophy of Science 73 (5): 730-42.

Wimsatt, W. C. 2012. "Robustness, Reliability, and Overdetermination (1981)." In Characterizing the Robustness of Science, ed. Léna Soler, 61-87. Dordrecht: Springer.

Wray, K. B. 2013. "The Future of the Structure of Scientific Revolutions." Topoi 32 (1): 75-79.

Zollman, K. J. 2007. "The Communication Structure of Epistemic Communities." Philosophy of Science 74 (5): 574-87.

- 2010. "The Epistemic Benefit of Transient Diversity." Erkenntnis 72 (1): 17-35.
2012. "Social Network Structure and the Achievement of Consensus." Politics, Philosophy and Economics 11 (1): 26-44.

. 2013. "Network Epistemology: Communication in Epistemic Communities." Philosophy Compass 8 (1): 15-27. 